Max-Planck-Institut für demografische Forschung Max Planck Institute for Demographic Research Konrad-Zuse-Strasse 1 - D-18057 Rostock · GERMANY

Tel +49 (0) 3812081 - 0; Fax +49 (0) 3812081 - 202;

http://www.demogr.mpg.de

MPIDR WORKING PAPER WP 2003-041

DECEMBER 2003

\title{
Modeling Failure (Mortality) Rate with a Change Point
}

M.S. Finkelstein (FinkelM@sci.uovs.ac.za)

This working paper has been approved for release by: James W. Vaupel (jwv@ demogr.mpg.de)

Head of the Laboratory of Survival and Longevity.

(C) Copyright is held by the authors.

Working papers of the Max Planck Institute for Demographic Research receive only limited review. Views or opinions expressed in working papers are attributable to the authors and do not necessarily reflect those of the Institute. 


\title{
Modeling Failure (Mortality) Rate with a Change Point
}

\author{
M.S. Finkelstein \\ Department of Mathematical Statistics \\ University of the Free State \\ PO Box 339, 9300 Bloemfontein, Republic of South Africa \\ (e-mail: FinkelM@sci.uovs.ac.za) \\ and \\ The Max Planck Institute for Demographic Research, \\ Rostock, Germany
}

Draft: 3 December 2003

\begin{abstract}
Simple models for the failure (mortality) rate change point are considered. The relationship with the mean residual lifetime function change point problem is discussed. It is shown that when the change point is random, the observed failure (mortality) rate can be obtained via a specific mixture of lifetime distributions. The shape of the observed failure (mortality) rate is analyzed and the corresponding simple but meaningful example is considered.
\end{abstract}

Keywords: Change point; Failure rate; Mortality rate; Mean residual lifetime function; Mixture of distributions.

\section{INTRODUCTION.}

In lifetime data analyses it is often reasonable to assume that early failures obey one distribution (sometimes it is called the "infant mortality" cumulative distribution function (Cdf)), whereas after some time another distribution usually with a smaller failure rate comes into play. It is well known that the mortality rate of humans is described by the exponentially increasing Gompertz curve for adults and decreases in the earlier part of life. Alternatively, an engineering device, starting its performance at some small level of electrical load can experience an increase of this load at some instance of time $\mathrm{z}$, or other parameters, characterizing the regime of functioning are changed at $z$. Most often, a change in the original pattern of the failure (mortality) rate is caused by some external factors (e.g., a change in environment). Therefore, the corresponding models should capture this combined pattern of the failure (mortality) rate. Considering the simplest models of this kind is the topic of this note. We discuss here only some general initial approaches to be specified in further studies.

Numerous demographic studies show that mortality rates of humans at all ages are consistently declining with chronological time (see [10] for recent data on the increase in life expectancy, for instance) at least for the last two centuries. On the other hand, along with these gradual processes, populations can experience change points due to positive or negative 'environmental' influences. The implementation of the better healthcare in the former East Germany after the reunification can be considered as an example of such a positive influence [13], whereas the demographic situation during the transitional period in Russia after the collapse of the USSR shows a negative im- 
pact on mortality rates. It is clear that the change point in the mortality rate can take place in both of these cases and the corresponding analysis can help to understand the true pattern of the mortality curve.

The concept of a random change point seems to be more natural in engineering applications than in demography. However, unobserved time of a change point in the mortality rate can be also encountered in population studies. On the other hand, this concept is reasonable for forecasting future mortality rates, when the change point is likely to occur, but its time is unknown.

We discuss in this note some new models for the failure (mortality) rate and the mean remaining lifetime (life expectancy) change point analysis. The corresponding general approaches are common to a certain extent for the demographic mortality studies and for the failure analysis of engineering systems as well. For the sake of presentation we shall use reliability notation $\lambda(t)$ for the failure rate, having also in mind demographic and actuarial notation $\mu(t)$ for the mortality rate (force of mortality). In order to keep this analogy as close as possible we, in fact, assume the cohort survivorship approach in the mortality rate modeling. In this case all survivors experience the change point at the same age. Generalization to the period survivorship with age specific change points for survivors is the topic for the future research.

General theoretical models to be considered can help to analyze the shape of the failure (mortality) rate, which is important for the failure (death) time analysis. It will be shown, for instance, that the random change point can be interpreted as an additional source of frailty in a population [14], which eventually bends down the mortality curve and exhibits an effect of "the weakest populations are dying out first" [4].

In Section 2 two main models for the failure (mortality) rate with a change point and the corresponding Cdfs are formulated. Section 3 is devoted to the relation between the change points in the failure (mortality) rate and the mean remaining lifetime (life expectancy at a given instant of time). In sections 4 and 5 the failure (mortality) rate with a random change point is defined and its shape is analyzed. Section 6 is devoted to a brief discussion. We also defer the discussion of inferential aspects of the change point analysis to future publications.

\section{MODELS}

Consider an object, which starts its lifecycle at $t=0$ in some environment (e.g., load, stress, or lifestyle, living conditions), which has been changed at $t=z$. The simplest failure rate model with a change point of this kind can be defined directly via the failure rates [10]:

$$
\lambda(t, z)=\lambda_{1}(t) I(t<z)+\lambda_{2}(t) I(t \geq z) ; t \geq 0,
$$

where $\lambda_{1}(t)$ is the failure rate before the change point and $\lambda_{2}(t)$ is the failure rate after it; $\lambda(t, z)$ is the 'combined' failure rate, and $I(t<z), I(t \geq z)$ are the corresponding indicators:

$$
I(t<z)=\left\{\begin{array}{ll}
1, & t<z \\
0, & t \geq z
\end{array}, \quad I(t \geq z)=\left\{\begin{array}{ll}
0, & t<z \\
1, & t \geq z
\end{array} .\right.\right.
$$

It is clear that formally equation (1) can be also written via the proportional hazards (PH) model:

$$
\lambda(t, z)=\theta(t, z) \lambda_{1}(t)
$$


where $\theta(t, z)=1$ for $t<z$ and $\theta(t, z)=\lambda_{2}(t) / \lambda_{1}(t)$ for $t \geq z$. This interpretation can be helpful, as the PH model usually reasonably describes the impact of a changing environment.

Denote the Cdfs which correspond to $\lambda_{1}(t), \lambda_{2}(t)$ and $\lambda(t, z)$ by $F_{1}(t), F_{2}(t)$ and $F(t, z)$, respectively. It is easy to see that relation (1) leads to the following equation for the survival function $\bar{F}(t, z) \equiv 1-F(t, z)$ :

$$
\bar{F}(t, z)=\bar{F}_{1}(t) I(t<z)+\bar{F}_{1}(z) \frac{\bar{F}_{2}(t)}{\bar{F}_{2}(z)} I(t \geq z),
$$

where $\bar{F}_{2}(t) / \bar{F}_{2}(z)=\exp \left\{-\int_{z}^{t} \lambda_{2}(u) d u\right\}$ is the conditional survival probability in $[z, t)$ for an object with the Cdf $F_{2}(t)$.

Relation (1) sets a change point model when $z$ is fixed or when the failure rate after the change point does not depend on z. It is clear that in this case one cannot obtain information on the location of the change point by observing the failure rate after the change point (which means: observing failures of an object). In some instances, however, the failure rate after the change point depends on $z$. Therefore, relation (1) can be generalized to

$$
\lambda(t, z)=\lambda_{1}(t) I(t<z)+\lambda_{2}(t, z) I(t \geq z) ; t \geq 0
$$

Equation (2) is also valid for this generalization, which can be seen after substitution $\bar{F}_{2}(t) / \bar{F}_{2}(z)$ by $\bar{F}_{2}(t, z) / \bar{F}_{2}(z, z)$, where $F_{2}(t, z)$ is defined by the failure rate $\lambda_{2}(t, z)$ in a usual way.

Example 1. a. Consider an object's lifetime in some baseline regime described by the Cdf $F_{1}(t)$ with the failure rate $\lambda_{1}(t)$. At $t=z$, the regime is switched on to a more severe one, characterized by a larger value of the failure rate: $\lambda_{2}(t) \geq \lambda_{1}(t)$. It is clear that this setting is described by the model (1).

b. Assume now that the change point is defined by the change (e.g., increase) in the derivative (slope) of the failure rate: $\lambda_{2}(z)=\lambda_{1}(z) ;\left.\lambda_{2}^{\prime}(t)\right|_{t=z}>\left.\lambda_{1}^{\prime}(t)\right|_{t=z}$ For instance, we can consider linear failure rates with $\lambda_{2}>\lambda_{1}>0$ :

$$
\begin{gathered}
\lambda_{1}(t)=\lambda_{1} t, \quad 0 \leq t<z, \\
\lambda_{2}(t, z)=\lambda_{2} t-\left(\lambda_{2}-\lambda_{1}\right) z, \quad t \geq z
\end{gathered}
$$

and this is, clearly, the second, more general model.

c. The mortality rate of the Gompertz distribution is given by the following relation: $\mu(t)=a \exp \{b t\} ; a>0, b>0$. A pure jump to a larger level can be modeled by $a^{\prime} \exp \{b t\}$, where $a^{\prime}>a$, whereas the change point in the slope similar to the previous case can be defined as:

$$
\begin{gathered}
\mu_{1}(t)=a \exp \{b t\}, \quad 0 \leq t<z, \\
\mu_{2}(t)=a \exp \left\{\left(b-b^{\prime}\right) z\right\} \exp \left\{b^{\prime} t\right\}, \quad t \geq z ; \quad b^{\prime}>b .
\end{gathered}
$$

Remark 1. This example illustrates the fact that the change point in the failure rate is usually characterized either by the jump in $\partial \lambda(t, z) /\left.\partial t\right|_{t=z}$ or by a jump in $\lambda(t, z)$ at $t=z$ (the latter can be also combined with the jump in the slope). In fact, the smooth 
case also exists, when there are no jumps in the function and its derivative, but there is a 'substantial change' in $\partial \lambda(t, z) / \partial t$ in the neighborhood of $t=z$. A bathtub shape of the failure rate, when initially the failure rate decreases, reaches a single minimum and then increases (or upside-down bathtub shape of the failure rate), illustrates this possibility.

\section{CHANGE POINT IN MRL}

In many instances analysis of the failure rate goes along with analysis of the mean remaining lifetime (MRL) function. Therefore, change points in the MRL function are also often of interest, although defining the change point via the failure rate concept is more traditional. The MRL change point concept was not practically explored in the literature so far, although we think it can present a reasonable and a 'more weak' alternative. The following question arises in this respect: does the change point in the failure rate always lead to the change point in the MRL at the same point? An immediate answer is obviously "no", whereas the more detailed considerations are presented in the rest of this section.

Let $X_{t}$ denotes the remaining lifetime (at time $t$ ) of an object with a governing Cdf $F(x)$ and a failure rate $\lambda(x)$. Then, in accordance with a standard definition [6,9], the MRL function $m(t)$ (life expectancy at time $t$ ) is given by:

$$
m(t) \equiv E\left[X_{t}\right]=\frac{\int_{t}^{\infty} \bar{F}(u) d u}{\bar{F}(t)}=\int_{0}^{\infty} \exp \left\{-\int_{t}^{x+t} \lambda(u) d u\right\} d x .
$$

Assume that $m(t)$ is differentiable. By differentiating the quotient in (4) the following important relation can be easily obtained:

$$
m^{\prime}(t)=\frac{\lambda(t) \int_{t}^{\infty} \bar{F}(u) d u-\bar{F}(t)}{\bar{F}(t)}=\lambda(t) m(t)-1
$$

or, equivalently [5]:

$$
\lambda(t)=\frac{m^{\prime}(t)+1}{m(t)} .
$$

Equation (6), in fact, defines the relationship between these two important functions. For exponential distribution: $\lambda=1 / \mathrm{m}$.

It follows immediately from definition (4) that $m(t)$ is a continuous function for an absolutely continuous $F(x)$ and that the jumps in the failure rate do not lead to the jumps in the MRL. Analyzing relation (6), it is easy to conclude that the jump in $\lambda(t)$ at $t=z$ corresponds to the jump in $m^{\prime}(t)$ at the same point.

Example 2. Consider the specific case of the model (1): $\lambda_{1}(t)=\lambda_{1} ; \lambda_{2}(t)=\lambda_{2}$. It is easy to see integrating by parts that: 


$$
\begin{aligned}
m(t) & =\left\{\begin{array}{l}
{\left[-(z-t) \exp \left\{-\lambda_{1}(z-t)\right\}+\frac{1}{\lambda_{1}}\left[1-\exp \left\{-\lambda_{1}(z-t)\right\}\right]\right.} \\
+\exp \left\{-\lambda_{1}(z-t)\right\}\left[(z-t)+\frac{1}{\lambda_{2}}\right]
\end{array}\right\} I(t<z)+\frac{1}{\lambda_{2}} I(t \geq z) \\
& =\left\{\frac{1}{\lambda_{1}}\left[1-\exp \left\{-\lambda_{1}(z-t)\right\}\right]+\frac{1}{\lambda_{2}} \exp \left\{-\lambda_{1}(z-t)\right\}\right\} I(t<z)+\frac{1}{\lambda_{2}} I(t \geq z)
\end{aligned}
$$

and

$$
\left.m^{\prime}(t)\right|_{t=-z}=-1+\frac{\lambda_{1}}{\lambda_{2}} ;\left.\quad m^{\prime}(t)\right|_{t=+z}=0
$$

where $\left.m^{\prime}(t)\right|_{t=-z}\left(\left.m^{\prime}(t)\right|_{t==_{+} z}\right)$ denote the value of the derivative with respect to $t$ just before (after) $t=z$. Thus, the jump $\lambda_{2}-\lambda_{1}$ in $\lambda(t)$ leads to the jump $1-\frac{\lambda_{1}}{\lambda_{2}}$ in $m^{\prime}(t)$ at $t=z$.

It is usually more difficult to define the change point for the MRL in the "no-jump situation' (see the Remark 1). It is worth mentioning that the change points in the failure rate and in the MRL (if any) do not coincide in this case. The following result illustrates this assertion and describes the relation between the shapes of $\lambda(t)$ and $m(t)$, when $\lambda(t)$ has a bathtub shape[5,7,9]:

Let $\lambda(t)$ be a differentiable bathtub failure rate.

(i) If

$$
m^{\prime}(0)=\lambda(0) m(0)-1<0
$$

then $m(t)$ is decreasing in $[0, \infty)$.

(ii) If $m^{\prime}(0)>0$, then $m(t)$ has an upside-down bathtub shape.

The symmetric result is valid for the upside-down bathtub failure rate. Thus, the change point in the failure rate in this case does not necessarily lead to the change point in $m(t)$.

\section{RANDOM CHANGE POINT}

In many instances the change point is unobserved or, alternatively, is random. This means that we know $\lambda_{1}(t)$ and $\lambda_{2}(t)$ (or the functional form of $\lambda_{2}(t, z)$ ), but we do not know z. The resulting (observed) failure rate $\lambda(t)$ is of a prime interest for this setting.

Consider the model (1). The forthcoming reasoning can be easily applied to the model (3) as well. Denote the random change point variable by $Z$ and let $\pi(z)$ be its probability density function (pdf) with support in $[0, \infty)$. Then the corresponding hazard rate process (random failure rate) [6] is defined as

$$
\lambda(t, Z)=\lambda_{1}(t) I(t<Z)+\lambda_{2}(t) I(t \geq Z) .
$$

It is clear that the lifetime Cdf of an object operating in an environment with a random change point can be modeled by the corresponding mixture: 


$$
F(t)=E[F(t, Z)]=\int_{0}^{\infty} F(t, z) \pi(z) d z .
$$

The mixture failure rate for the general case is defined by.

$$
\lambda(t)=\int_{0}^{\infty} \lambda(t, z) \pi(z \mid t) d z
$$

where the conditional pdf $\pi(z \mid t)$ is $[4,8,11]$ :

$$
\pi(z \mid t)=\frac{\pi(z) \bar{F}(t, z)}{\int_{0}^{\infty} \bar{F}(t, z) \pi(z) d z} .
$$

Applying these relations to models under consideration and taking into account relation (2):

$$
\pi(z \mid t)=\frac{\pi(z)}{\int_{0}^{\infty} \bar{F}(t, z) \pi(z) d z} \begin{cases}\bar{F}_{1}(t), & t<z \\ \frac{F_{1}(z)}{\bar{F}_{2}(z)} \bar{F}_{2}(t), & t \geq z\end{cases}
$$

Eventually:

$$
\lambda(t)=\frac{\lambda_{1}(t) \bar{F}_{1}(t) \int_{t}^{\infty} \pi(z) d z+\lambda_{2}(t) \bar{F}_{2}(t) \int_{0}^{t} \frac{\bar{F}_{1}(z)}{\bar{F}_{2}(z)} \pi(z) d z}{\bar{F}_{1}(t) \int_{t}^{\infty} \pi(z) d z+\bar{F}_{2}(t) \int_{0}^{t} \frac{\bar{F}_{1}(z)}{\bar{F}_{2}(z)} \pi(z) d z} .
$$

The similar result is valid for the model (3):

$$
\lambda(t)=\frac{\lambda_{1}(t) \bar{F}_{1}(t) \int_{t}^{\infty} \pi(z) d z+\int_{0}^{t} \lambda_{2}(t, z) \bar{F}_{2}(t, z) \frac{\bar{F}_{1}(z)}{\bar{F}_{2}(z, z)} \pi(z) d z}{\bar{F}_{1}(t) \int_{t}^{\infty} \pi(z) d z+\int_{0}^{t} \bar{F}_{2}(t, z) \frac{\bar{F}_{1}(z)}{\bar{F}_{2}(z, z)} \pi(z) d z}
$$

Relations (11) and (12) can be used for describing the shape of the observed failure rate, which is usually rather cumbersome and only the simplest settings can be analyzed directly.

\section{THE SHAPE OF THE FAULURE (MORTALITY) RATE}

It follows from formulas (11) and (12) that the shape of the observed mortality rate $\lambda(t)$ differs from the shapes of $\lambda_{1}(t)$ and $\lambda_{2}(t)$. We shall discuss some general properties of the shape of the mortality rate curve for a simpler and more frequently used model (1)-(2), (11). The analysis for model (3) is more complicated and usually should be performed using numerical methods.

It is interesting to find, at first, when the following asymptotic relation:

$$
\lambda(t) \rightarrow \lambda_{2}(t)
$$

holds as $t \rightarrow \infty[3]$. By relation (13) we mean:

$$
\lambda(t)=\lambda_{2}(t)(1+o(1)), t \rightarrow \infty
$$

When $\lambda_{2}(t)$ is bounded: $\lambda_{2}(t) \leq C>0 ; \forall t \geq 0$, relation (14) is equivalent to $\left|\lambda(t)-\lambda_{2}(t)\right| \rightarrow 0$ as $t \rightarrow \infty$. Denote: 
Then equation (11) turns to

$$
\alpha(t) \equiv \frac{\bar{F}_{1}(t) \int_{t}^{\infty} \pi(z) d z}{\bar{F}_{2}(t) \int_{0}^{t} \frac{\bar{F}_{1}(z)}{\bar{F}_{2}(z)} \pi(z) d z} .
$$

$$
\lambda(t)=\frac{\alpha(t) \lambda_{1}(t)+\lambda_{2}(t)}{\alpha(t)+1} .
$$

Consider, as in Example 1, the case of the more severe regime after the change point, when $\lambda_{2}(t)>\lambda_{1}(t), \forall t>0$ (the case or a lighter regime after the change point is analyzed in a similar way). Therefore, if $\lambda_{1}(t) \rightarrow \infty$, the evident sufficient condition for (14) is

$$
\alpha(t) \lambda_{1}(t) \rightarrow 0, t \rightarrow \infty
$$

On the other hand, if $\lambda_{1}(t)$ is bounded, then $\alpha(t) \rightarrow 0$ guarantees that $\left|\lambda(t)-\lambda_{2}(t)\right| \rightarrow 0$ as $t \rightarrow \infty$. Monotonicity properties of $\lambda(t)$ for the general case can be studied by considering $\lambda^{\prime}(t)$, but the corresponding expression is rather cumbersome and explicit results can be obtained only for specific cases.

It follows from equation (15) that under given assumptions $\lambda(t)<\lambda_{2}(t), \forall t>0$. On the other hand, even for the bounded rates the distance $\lambda_{2}(t)-\lambda(t)$ does not necessarily tend to 0 . This interesting fact is illustrated by the following meaningful example.

Example 3. The direct analysis of the shape of $\lambda(t)$ for the following setting is of interest. Let: $\lambda_{1}(t)=\lambda_{1} ; \lambda_{2}(t)=\lambda_{2}$ and $\pi(z)$ be also exponential: $\pi(z)=\lambda_{s} \exp \left\{-\lambda_{s} z\right\}$. Using relation (11), after simple rearrangements:

$$
\lambda(t)=\frac{\lambda_{1}+\frac{\lambda_{2} \lambda_{s}}{\lambda_{2}-\lambda_{1}-\lambda_{s}}\left(1-\exp \left\{-\left(\lambda_{2}-\lambda_{1}-\lambda_{s}\right\} t\right)\right.}{1+\frac{\lambda_{s}}{\lambda_{2}-\lambda_{1}-\lambda_{s}}\left(1-\exp \left\{-\left(\lambda_{2}-\lambda_{1}-\lambda_{s}\right\} t\right)\right.}
$$

a. Let $\lambda_{2}>\lambda_{1}+\lambda_{s}$. Then from relation (17)

$$
\lim _{t \rightarrow \infty} \lambda(t)=\lambda_{1}+\lambda_{s}
$$

Thus, in this case $\lambda_{1}<\lambda(\infty)<\lambda_{2}$ and for sufficiently small $\lambda_{s}$ the pdf $\pi(z)$ gives more "weight" to smaller values of $t$, where $\lambda(t)=\lambda_{1}$ with a larger probability. Differentiating the right hand side of (17), it can be easily shown that $\lambda^{\prime}(t)>0, \forall t \geq 0$, which means that $\lambda(t)$ monotonically increases from the level $\lambda_{1}$ to the level $\lambda_{1}+\lambda_{s}$ as $t \rightarrow \infty$. It can be easily checked that condition $\alpha(t) \rightarrow 0$ as $t \rightarrow \infty$ does not hold in this case.

b. Let $\lambda_{1}<\lambda_{2}<\lambda_{1}+\lambda_{s}$. Then from relation (17):

$$
\lim _{t \rightarrow \infty} \lambda(t)=\lambda_{2}
$$

as the probability distribution in this case gives more weight to larger values of $t$, where $\lambda(t)=\lambda_{2}$ with a larger probability. It can be also checked that the general condition $\alpha(t) \rightarrow 0$ as $t \rightarrow \infty$ holds in this case. 
c. Finally, the limit (19) also holds for $\lambda_{2}<\lambda_{1}$ (the improved mortality rates after the change point, which is characteristic for the current demographic practice). This fact can be easily seen from equation (17). Another interpretation of the shape of $\lambda(t)$ is due to the well-known property: the mixture of Cdfs with decreasing failure rates is the Cdf with the decreasing failure rate as well [1,2]. It is worth noting, that the preservation of the increasing failure rate property under operation of mixing was also observed in specific cases a. and b. of this example, whereas it is not true for the general case of increasing rates [4].

Remark 2. In fact, it is rather surprising that $\lim _{t \rightarrow \infty} \lambda(t)$ in this example depends on the relationship between $\lambda_{1}, \lambda_{2}$ and $\lambda_{s}$.

Remark 3. The random change point introduces specific heterogeneity in the model and the effect of the mortality rate bending down with age can be explained also by this effect.

\section{CONCLUDING REMARKS}

We have considered two rather general models for the failure (mortality) rate change point and have analyzed the shape of the observed rate $\lambda(t)$, when the change point is random. It turns out that the random change point model is a specific case of mixing and some general results on the failure rates of mixtures can be applied. For instance, it is well known that the class of Cdfs with decreasing failure rates is closed under the operation of mixing. On the other hand, this operation is not closed for the class of Cdfs with increasing failure rates. However, the specific cases of Example 3 show that under additional assumptions this property can still hold.

It is natural to define the change point via the failure (mortality) rate, because it describes the direct effect of environment on a lifetime of an object. Due to relationship (6), the corresponding change point in the MRL function can be also analyzed. It was shown that under certain assumptions the MRL function does not have a change point (though $\lambda(t)$ has) and the jump in the failure rate corresponds to the jump in the derivative of the MRL function at the same instant of time.

As it was mentioned in section 1, we exploit the analogies between demographic and engineering applications to a full extent and assume the cohort survivorship approach in the mortality rate modeling. Generalization to the period survivorship with age specific change point for survivors and the corresponding inferential problems are the topics for the further research.

\section{References}

1. Barlow R., and Proschan F. (1975). Statistical Theory of Reliability and Life Testing. Probability Models, Holt, Rinehart and Winston, New-York

2. Barlow, R. E. (1985). A Bayes explanation of an apparent failure rate paradox. IEEE Trans. Reliability, R-34, 107-108.

3. Block, H. W. and Joe, H. (1997) Tail behavior of the failure rate functions of mixtures. Lifetime Data Analyses, 3: 269-288. 
4. Finkelstein, M.S. and Esaulova V. (2001) Modeling a failure rate for a mixture of distribution functions. Probability in the Engineering and Informational Sciences, 15: 383-400.

5. Finkelstein, M.S. (2002). On the shape of the mean residual life function. Applied Stochastic Models in Business and Industry, 18, 135-146

6. Finkelstein, M.S. (2003) Simple bounds for terminating Poisson and renewal processes. Journal of Statistical Planning and Inference, 113, 541-548

7. Gupta, R.C. and Akman, H.O. (1995). Mean residual life function for certain types of non-monotonic aging. Stochastic models, 11: 219-225.

8. Lynn, N. J. and Singpurwalla, N.D. (1997) Comment: "Burn-in" makes us feel good. Statistical Science, 12: 13-19.

9. Mi, J. (1995). Bathtub failure rate and upside-down bathtub mean residual life. IEEE Transactions on reliability, 44: 388-391.

10. Oeppen J., and Vaupel J.W. (2002). Broken limits to life expectancy. Science, 296, 1029-1031.

11. Patra, K., and Dey, D. (2002). A general class of change point and change curve modeling for lifetime data. Ann. Inst. Statist. Math. 54: 517-530.

12. Shaked, M., and Spizzichino, F. (2001). Mixtures and monotonicity of failure rate functions In: N. Balakrishnan and C.R. Rao, eds., Handbook of Statistics, 20, 185198, Elsevier, London.

13. Scholtz R., and Mair H. (2003). German unification and the plasticity of mortality at older ages. Working Paper of the Max Planck Institute for Demographic Research, 2003-031, http://www.demogr.mpg.de/

14. Vaupel, J.W., Manton K.G., and Stallard E. (1979). The impact of heterogeneity in individual frailty on the dynamics of mortality. Demography, 16, 439-454.

15. Yashin, A.I., Iachin, I., and Begun, A.S. (2000). Mortality modeling: a review. Mathematical Population Studies, 8, 305-332. 